Joseph - Arterial Inflammation and Atherosclerosis

\title{
Short-Term Changes in Arterial Inflammation Predict Long-Term Changes in \\ Atherosclerosis Progression
}

Philip Joseph (1,2)*, Amorina Ishai (1)*, Venkatesh Mani (3), David Kallend (4), James H.F. Rudd (5), Zahi A. Fayad (3)**, Ahmed Tawakol (1)**

(1) Harvard Medical School and Massachusetts General Hospital, Boston, MA, USA

(2) Population Health Research Institute, Department of Medicine, and Department of Radiology, McMaster University, Hamilton, ON, CAN

(3) Translational and Molecular Imaging Institute and Department of Radiology, Icahn School of Medicine at Mount Sinai School of Medicine, New York, NY, USA

(4) The Medicines Company, Parsippany, New Jersey

(5) Division of Cardiovascular Medicine, University of Cambridge, Cambridge, UK

*Designates co-primary authorship for the study

** Designates co-senior authorship for the study

Word Count: 6296

Corresponding Authors: Ahmed Tawakol M.D., Cardiac MR PET CT Program, Division of Cardiac Imaging, Massachusetts General Hospital and Harvard Medical School, Suite 400-165 Cambridge St, Boston MA 02114. Email: atawakol@mgh.harvard.edu, Fax: (617) 724-4152; Phone: (617) 726-0791 
Zahi Fayad, Ph.D., Hess CSM Building Floor TMII - 1st Floor Room Z.Fayad - Rm S1-104,

1470 Madison Avenue, New York, NY 10029, Email: zahi.fayad@mssm.edu, Tel: 212-241-

8452, Fax: 646-537-9693 


\section{Abstract:}

Purpose: It is not known if changes in arterial wall inflammation associate with subsequent changes in the rate of structural progression of atherosclerosis.

Methods: In this sub-study of the dal-PLAQUE clinical trial, multi-modal imaging was performed using 18-Fludeoxyglucose (FDG) positron emission tomography (PET, at 0 and 6 months) and magnetic resonance imaging (MRI, at 0 and 24 months). The primary objective was to determine whether increasing FDG uptake at 6-months predicted atherosclerosis progression on MRI at 2 years. Arterial inflammation was measured by the carotid FDG target to background ratio (TBR), and atherosclerotic plaque progression was defined as the percent change in carotid mean wall area (MWA) and mean wall thickness (MWT) on MRI between baseline and 24 months.

Results: The mean age of the population was 62.5 years, and 12 (28.6\%) were female. In participants with (vs. without) any increase in arterial inflammation over 6 months, both the long-term changes in MWT ( $\%$ change MWT: $17.49 \%$ vs. $1.74 \%, \mathrm{p}=0.038$ ) and MWA ( $\%$ change MWA: $25.50 \%$ vs. $3.59 \%, p=0.027$ ) were significantly greater. Results remained significant after adjusting for clinical and biochemical covariates. Individuals with no increase in arterial inflammation over 6 months had no significant structural progression of atherosclerosis over 24 months, as measured by MWT $(\mathrm{p}=0.616)$ or MWA $(\mathrm{p}=0.373)$.

Conclusions: Short-term changes in arterial inflammation are associated with long-term structural atherosclerosis progression. These data support the concept that therapies that reduce arterial inflammation may attenuate or halt progression of atherosclerosis. 
Key Words: Positron emission tomography, inflammation, atherosclerosis 


\section{Introduction:}

Atherosclerosis and its major clinical consequences, myocardial infarction and stroke, are leading causes of mortality and morbidity worldwide. Inflammation plays a crucial role in the development and progression of atherosclerosis, and can be non-invasively measured using 18-Fludeoxyglucose (FDG) positron emission tomography/computed tomography (PET/CT).1,2 FDG is a glucose analog that is retained within cells at a rate proportional to glucose dependent metabolism; and compared to other vascular cells, FDG uptake is avid in immune cells. In the vascular wall, this correlates strongly with atherosclerotic plaque burden, and macrophage infiltration. ${ }^{3}$ Rapid changes in FDG arterial uptake have been observed in humans in response to therapeutic interventions. However, there is limited data examining how these short-term changes in inflammatory activity in an arterial wall relate to subsequent atherosclerosis progession.

The dal-PLAQUE study was a phase $2 \mathrm{~b}$ double blind, randomized, placebocontrolled trial examining the effects of the cholesterol-ester-transfer-protein (CETP) modulator dalcetrapib on structural and inflammatory characteristics of arterial wall atherosclerosis by PET/CT over a 6 month period, and/or by magnetic resonance imaging [MRI] imaging over 24-months. ${ }^{4}$ Prior analysis of dal-PLAQUE identified a significant association between changes in carotid arterial inflammation over 6 months and enlargement of the total vessel area at 24 months. However, the total vessel area includes not only arterial wall, but also the lumen, and this observation could have simply reflected changes in the lumen, thus making it difficult to conclude whether these early inflammatory changes predicted atherosclerosis development. Indeed, there is limited data 
in humans examining the relationship between changes in arterial inflammation and atherosclerotic disease progression. Further, while dal-PLAQUE included an intervention (which did not significantly change arterial inflammation), it provides an opportunity to test the hypothesis that changes in arterial inflammation over time (regardless of the underlying treatment milieu) result in changes in plaque progression. Accordingly, the objective of this study is to determine whether short-term changes in arterial inflammation (measured by FDG-PET) predicted long-term changes atherosclerosis progression (measured as wall thickness and wall area by MRI).

\section{Methods:}

\subsection{Study design}

This was a prospective, sub-study of participants enrolled in the dal-PLAQUE clinical trial. The design of dal-PLAQUE has been described in detail in previous publications., ${ }^{4,5}$ Briefly, dal-PLAQUE enrolled 130 participants from 11 centers in the United States and Canada. Participants were randomized (1:1) to receive either dalcetrapib $600 \mathrm{mg} /$ day or placebo over a 24-month period. Participants could undergo 18-FDG-PET/CT to evaluate inflammation in the carotid arteries at 3 and 6 months; and/or black-blood MRI to assess anatomic indices of carotid plaque burden at 6-, 12- and 24-months (undergoing all imaging time points was not required). This analysis included 42 participants from dalPLAQUE who had serial FDG-PET/CTs at baseline and 6 months, and serial MRIs at baseline and two years. The study was approved by each participating center's institutional review committee, and all subjects provided informed consent. 


\subsection{Imaging by PET/CT}

Imaging protocols for FDG-PET/CT have been described in detail in previous publications.4, ${ }^{5}$ FDG-PET/CT imaging of the carotid arteries was performed using previously validated, reproducible imaging methods. ${ }^{6,7}$ Maximum standard uptake values (SUV) of FDG were measured, in axial orientation, along each carotid artery from $1 \mathrm{~cm}$ above to $2 \mathrm{~cm}$ below the bifurcation by manually drawing circular regions of interest that encompassed the vessel wall at 3-5 mm increments. Target-to-background ratios (TBR) were calculated for each segment by dividing each SUV value by the background venous FDG activity measured in the internal jugular veins. The TBR of the most diseased segment (MDS) was calculated as a mean of the three contiguous segments centered at the vessel segment with the highest maximum TBR, and was used as the primary measure of FDG uptake for this study.

\subsection{Imaging by MRI}

MRI imaging of the common carotid arteries was performed using previously validated methods, which included proton density-weighted, T1-weighted and T2-weighted darkblood turbo spin echo sequences to image the vessel walls, with additional phase contrast and time-of-flight sequences for better localization of carotid bifurcations.8, ${ }^{8} 9$ External and internal walls of the carotid arteries were manually traced, and vessel wall area and thickness were calculated as the primary markers of atherosclerosis burden for this analysis. 


\subsection{Statistical analysis}

The primary independent variable of interest was an increase in carotid arterial inflammation (i.e. increase in TBR) between baseline and 6-months. Percent changes in mean wall thickness (MWT) and mean wall area (MWA) of the carotid arteries were calculated by MRI between baseline and 24 months as the primary outcomes of interest.

The primary analysis compared whether increase FDG activity at 6 months was associated with significant difference in percent change in MWT by MRI at 24 months using linear regression. Further multivariable linear regression analyses were performed to assess whether these differences remained significant after adjusting for 1) demographic variables and 2) selected baseline clinical, biochemical or imaging variables. Potential covariates were selected based on their established relationship with atherosclerosis development in prior literature, and included demographic variables (e.g. age, sex), cardiovascular risk factors, history of coronary artery disease, and biochemical markers of inflammation (e.g. C-reactive protein (CRP), Interleukin-6 (IL-6) and plasminogen activator inhibitor-1 (PAI-1) activity). Of these inflammatory biomarkers, PAI-1 activity was previously shown to predict changes in MWA on MRI in a prior dal-PLAQUE analysis. ${ }^{10}$ Covariates significantly associated with MWT at 24 months on univariate analysis (at a threshold p-value of 0.05) were carried forward in the multivariable analysis. Similar analyses were performed using the outcome of percent change in MWA measured by MRI at 24 months. Sensitivity analyses were also performed using absolute changes in MWA and MWT. 
Several exploratory analyses were also performed. To account for changes in the PET signal that could be attributable to differences in arterial wall area, we further adjusted for mean wall area at 6 months in our multivariable analysis. We also examined whether increased FDG activity remained a significant predictor of plaque progression by MRI after adjusting for biochemical markers of inflammatory activity, measured by Creactive protein between baseline and 12 months. Also, in a subset of participants with FDG-PET imaging at 3 months, we examined whether arterial inflammation was associated with atherosclerotic progression by MRI at 2 years, and the correlation between the percent change in SUV activity from baseline to 3- and 6-months. The association between changes in arterial inflammation and atherosclerosis progression, both measured between baseline and 6-months, was also examined. The association between baseline arterial inflammation and change atherosclerosis progression on MRI at 24 months was also measured. In participants with the largest amount of atherosclerosis progression on MRI (defined as the highest tertile group) we examined whether there was evidence of greater changes in arterial inflammation compared to groups with less atherosclerosis progression (defined as the lowest and middle tertile). Finally, to examine to short-term structural changes impact long-term atherosclerosis progression, we compared changes in MWT (and MWA) on MRI at 6 months and 24 months. Analyses were performed using R (version 3.1.4) and a p-value of 0.05 was considered the threshold for statistical significance. 


\section{Results:}

\subsection{Baseline characteristics:}

Forty-two participants provided complete multi-modal imaging datasets (PET/CTs at 0 and 6 months, and MRIs at 0 and 24 months). Baseline characteristics of the study population are summarized in table 1 . The mean age of the population was 62.5 years, and 12 (28.6\%) were female. Thirty-five participants (83.3\%) had a prior history of cardiovascular disease, $14(33.3 \%)$ were diabetic, $30(71.4 \%)$ had a history of hypertension and $4(9.5 \%)$ were smokers. Compared to individuals in dal-PLAQUE who did not provide complet multimodality data, participants included in this analysis had a lower mean body mass index (BMI) and systolic blood pressure. Also, the baseline FDG target-to-background ratio on PET, and baseline mean wall thickness (MWT) and mean wall area (MWA) on MRI were lower in included participants.

\subsection{Atherosclerosis progression in participants with or without increased arterial inflammation}

Eighteen participants (42.8\%) manifested an increase in arterial inflammation measured by PET/CT between baseline and 6 months. Structural atherosclerosis progression measured by percent changes in MWT and MWA on MRI over 24 months are summarized in Table 2. In participants with an early increase in arterial inflammation (baseline to 6 months), structural atherosclerosis progression was also seen at 2 years. Conversely, in participants with no increased arterial inflammation at 6 months, no significant atherosclerosis progression was observed. 
In the univariate linear regression analysis, evidence of increased arterial inflammation at 6 months was associated with a significantly greater change in both MWT and MWA on MRI at 24 months (Table 3 and Figure 1a). In participants with increased arterial inflammation on PET over 6 months, the increase in MWT over 24 months was approximately $15.8 \%$ greater when compared to participants without increased arterial inflammation ( $p=0.038)$. Similarly, in participants with increased arterial inflammation at 6 months, the increase in MWA at 24 months was approximately 21\% greater $(p=0.027)$ (Table 3 and Figure 1b). These differences remained significant after adjusting for age, gender and treatment allocation; and for additional clinical, biochemical or imaging variables significantly associated with changes in MWT on univariate analyses (see supplementary table 1). Results also remained significant after adjusting for structural atherosclerosis measurements obtained at 6-months (i.e mean wall area on MRI; $p=0.04$ ). On the other hand, baselineFDG uptake was not significantly associated with percent change in MWA over 24 months either on univariate analysis ( $\beta=-21.01$ (SE 11.10), $p=$ 0.07) or after adjusting for demographic variables and treatment allocation $(\beta=-22.9$ (SE 13.6), $\mathrm{p}=0.10$ ). Furthermore, using covariate adjustments specified in dalPLAQUE, we evaluated the effect of dalcetrapib on arterial inflammation (carotid atherosclerosis) ${ }^{4}$. Compared to placebo, treatment with dalcetrapib did not significantly reduce carotid inflammation over 6 months ( $-5.1 \%$ [SE 4.1] change in TBR, $p=0.22)$. It should be noted, however, that while the findings are consistent with the non-significant trend seen in the parent study, this sub-study analysis had limited power to assess the treatment effect.

In sensitivity analyses, we evaluated the association between increased arterial inflammation at 6 months and absolute changes in MWT and MWA at 24 months. Results 
are summarized in supplementary table 2 , and were consistent with our primary observations. Both associations trended towards significance in univariate analyses, and were statistically significant after adjusting for other significant predictors (derived from the primary analysis). Finally, we observed that increased arterial inflammation was also associated with short-term atherosclerosis progression (also measured at 6-months) (Supplementary table 3).

\subsection{Changes in Inflammatory Biomarkers and Atherosclerosis Progression by MRI:}

Over 24 months, no significant positive associations between changes in inflammatory biomarkers and either changes in MWT or MWA observed (Supplementary Table 4). There was a small inverse association between changes in SVCAM (Vascular cell adhesion molecule) and both MWT and MWA. A trend towards a significant inverse association was also observed with changes in sICAM (Intercellular adhesion molecule) and both MWT and MWA. Changes in arterial inflammation measured by PET remained significantly associated with atherosclerosis progression after adjusting for changes in either $\operatorname{sICAM}(\mathrm{p}=0.030)$ or sVCAM $(p=0.022)$. Changes in arterial inflammation also remained significant after correcting for changes in CRP ( $\mathrm{p}=0.047)$.

\subsection{Relationship between changes in arterial inflammation at 3 months and atherosclerosis progression at 24 months}

Three month PET studies were available in 41 participants. Changes in FDG uptake from baseline to 3 months correlated moderately with changes from baseline to 6 months (Pearson's Correlation $=0.74, \mathrm{p}<0.001)$. However, we did not observe any significant 
associations between evidence of increased arterial inflammation at 3 months and percent change in either MWT (p-value $=0.19)$ or MWA $(p$-value $=0.16)$ at 24 months.

\subsection{Mean Changes in Carotid Arterial Inflammation in Participants with the Highest Rates of Progression of Atherosclerosis on MRI}

In participants with the highest rate of atherosclerosis progression (defined as the highest tertile of change in MWT at 24 months), mean changes in TBR were significantly higher when compared to groups with evidence of less atherosclerosis progression (defined as the lower two tertiles $)(0.09[S D=0.32]$ versus $-0.16[S D=0.34], p=0.026$, Figure 2a). Similar results were obtained when participants were grouped into tertiles based on changes in MWA $(0.11[S D=0.28]$ for highest tertile versus -0.17 [SD $=0.35]$ for lowest two tertiles, $\mathrm{p}=$ 0.014 , Figure $2 b)$.

\subsection{Progression of atherosclerosis at 6 months compared to 24 months measured by MRI}

Thirteen of 35 participants (35.4\%) had evidence of increasing wall thickness on MRI at 6 months. Evidence of increasing MWT and 6-months was also associated with a greater rate of atherosclerosis progression at 24 months (see supplementary table 5). Change in MWT at 6 months as a continuous variable was highly correlated with change in MWT at 24months (Pearson's correlation $=0.64, \mathrm{p}<0.001$ ). Results using MWA were consistent with those of MWT (see supplementary table 5). 


\section{Discussion:}

In this sub-study of dal-PLAQUE, we found that short-term changes in arterial inflammation predict long-term structural atherosclerosis progression. Participants with evidence of increased carotid inflammation measured by FDG-PET over 6-months had greater progression in carotid artery MWT and MWA on MRI at 2 years. Also, in those without evidence of increased arterial inflammation, no significant atherosclerosis progression was observed.

These findings provide additional mechanistic insights into the consequences of arterial inflammation. Prior histologic, cross-sectional studies of carotid arterial imaging have shown that arterial inflammation measured by FDG-PET closely correlates with macrophage infiltration within the atheroma. 7,11 Furthermore, carotid inflammation measured by FDG-PET has been shown to precede the development of calcification within the index artery measured by computed tomography..$^{12}$ While these studies examining arterial inflammation cross-sectionally have increased our understanding of how arterial inflammation relates to both plaque activity and the progression of atherosclerosis, studies had yet to determine how changes in arterial inflammatory activity impact the progression of atherosclerosis.

Our study extends upon this current knowledge and provides two important pathophysiologic findings. First, changes in vascular inflammation observed over a short period appear to have both short and long-term consequences on atherosclerosis progression. Second, in those without evidence of increased inflammation, there were no significant changes in MWT or MWA observed, suggesting that atherosclerosis progression 
can be substantially mitigated when inflammation is reduced. This is also supported by our observations that in participants with the highest rates of atherosclerosis progression on MRI, changes in arterial inflammation were significantly higher when compared to groups with evidence of less atherosclerosis progression. The critical threshold delineating those with or without increased inflammation may be of added importance, as we observed stronger associations when participants were dichotomized into these groups compared to examining arterial inflammation as a continuous measure (Supplementary appendix, section 1).

Previous studies have shown that markers of systemic inflammation (e.g. CRP) also strongly predict plaque progression and cardiovascular events. ${ }^{13}$ Interestingly, in our exploratory analyses, we did not observe that changes in inflammatory biomarkers positively corresponded to atherosclerosis progression over the same period of time. These findings suggest that directly measuring inflammatory changes at the local, vascular level have a greater significance for identifying atherosclerosis progression compared to more general inflammatory biomarkers. However, whether this observation is generalizable to larger populations, and how this related to clinical outcomes, requires further study.

We did not observe an association between baseline FDG arterial uptake on PET and subsequent atherosclerosis progression, a finding that conflicts with prior studies. ${ }^{12}$ However, the current study included an intervention; the arterial milue indeed changed over the course of this study, as evidenced by the fact that a substantial percentage of individuals experienced changes in arterial inflammation. Thus comparisons between baseline arterial inflammatory measures and atherosclerosis progression should be 
interpreted with caution, and should not suggest that cross-sectional PET measures of inflammation are not valuable. Indeed, in individuals who are not enrolled in interventional studies, the baseline arterial signal associates with both future plaque progression as well as future clinical events. ${ }^{12,14}$ Prior clinical outcome studies that have consistently demonstrated a strong association between baseline arterial inflammation and subsequent cardiovascular events. In 518 patients without a history of active cancer or CVD, we observed that baseline arterial FDG activity strongly predicted future adverse cardiovascular events at a mean follow up of 4.2 years, and provided additional predictive value above clinical factors. ${ }^{14}$ Similarly, in a study of 64 patients with significant symptomatic carotid stenosis that underwent PET imaging, Marnane et al. observed a significant association between carotid arterial inflammation and recurrent ipsilateral stroke. ${ }^{15}$ Our findings provide additional insight to these clinical observations, suggesting that altherosclerosis development is also partly related to the consequences of persistent inflammation.

The results of our study further support the use of FDG-PET as a non-invasive imaging modality to predict the clinical impact of novel therapeutic agents on future cardiovascular outcomes. For example, using FDG-PET, a dose-dependent reduction in arterial inflammation has been observed over a 12-week period in response to the initiation of hydroxymethyl glutaryl coenzyme A (HMG-CoA) reductase inhibitor (statin) therapy, which is similar to the dose-dependent reductions in cardiovascular outcomes observed in several large clinical trials. ${ }^{16-18}$ Conversely, in the overall dal-PLAQUE study, CETP modulation did not significantly impact changes arterial inflammation when compared to placebo within any of the predefined PET endpoints (though was nearly 
significant in post-hoc analyses).${ }^{4}$ Similarly, dalcetrapib did not reduce vascular events in clinical outcome trials. ${ }^{4,}{ }^{19}$ Similarly, Inhibition of lipoprotein-associated phospholipase A2 (Lp-PLA2), fails to reduce atherosclerotic inflammation, ${ }^{20}$ a finding that is consistent with the lack of clinical efficacy of Lp-PLA2 antagonists. ${ }^{20-22}$ Most recently, P38 MAP Kinase antagonists have been found to be ineffective in reducing any of the pre-defined PET endointsand have similarly been found to be ineffective in reducing clinical events. ${ }^{23-25}$ Thus for all classes of drugs for which there is PET imaging and clinical endpoint trials data, the pre-defined PET findings have been concordant with the clinical endpoint findings.

Finally, we explored whether even earlier changes in FDG-PET uptake could predict atherosclerosis progression, and although we found 3-month changes in arterial inflammation correlated well with later, 6 month changes in the inflammatory signal, 3month changes themselves were not predictive of atherosclerosis progression. Ultimately, determining the optimal period for evaluate changes in arterial FDG uptake to determine the efficacy of novel therapeutic agents will depend on several factors, including the pharmacological properties of the treatments under investigation.

As anticipated, we also observed that increasing WMT and WMA measured by MRI over 6 months was also associated with a higher rate of atherosclerosis progression over 24 months. The consequences of these short-term structural changes to the arterial wall are complementary to our PET findings, and it is likely that these processes are interrelated, with biologically active atherosclerotic lesions also demonstrating early structural changes that both predict long-term atherosclerosis progression. Current cardiovascular pharmacologic therapies are widely given to patients with established CVD 
or at high risk for $\mathrm{CV}$ events due to their low cost, and highly favorable benefit-to-risk profiles. ${ }^{26,27}$ However, as future pharmacologic therapies develop, there may be a greater justification for measuring arterial inflammation with PET. ${ }^{20-22}$ Currently, antiinflammatory strategies for reducing CVD risk are being evaluated using both PET/CT imaging and clinical endpoint assessments (including colchicine, methotrexate and interleukin-1 inhibition). ${ }^{28}$ If these are shown to concurrently improve arterial inflammation as well as clinical outcomes, such studies would provide additional data to support the hypothesis that targetting arterial inflammation per se is clinically beneficial; and move future Phase 1 studies of pharmacologic agents that target inflammation to Phase III clinical endpoint trials with greater confidence based on FDG-PET results. Also, it is possible that imaging arterial inflammation could be one day employed to identify which patients are most likely to benefit from novel anti-inflammatory drugs (particularly expensive drugs and/or those with substantial side-effects). In such a case, individuals with significant arterial inflammatory disease at baseline, and/or who have demonstrable improvements after treatment would be targeted to receive long-term treatment. This personalized approach to CVD management would be similar to strategies currently used in oncology, where FDG-PET imaging is used for cancer staging , as well as post-induction re-staging of disease burden to guide further management decisions. ${ }^{29-31}$ However, prior to any clinical implementation, further studies are needed to determine whether the benefits of this approach would outweigh potential harms (e.g. radiation exposure), and whether it would be cost effective.

The main strength of our paper is that all PET and MRI studies were performed in the context of a clinical trial, where imaging techniques were standardized across sites and 
results were centrally interpreted. This significantly reduces the risk of biases related to variability in imaging procedures. Some limitations of our study also warrant consideration. Firstly, our primary outcome measures of MRI MWT and MWA represent non-invasive, surrogate markers of atherosclerosis development, since more invasive approaches were not employed in dal-PLAQUE. However, findings of atherosclerosis on MRI been shown to correlate well with observations of plaque burden by histology or other prognostic imaging modalities. For our analysis, serial PET and MRI images were available in approximately one-third of the participants randomised in dal-PLAQUE. Larger studies are needed to replicate these observations and demonstrate their generalizability in broader patient populations.

In conclusion, our observations demonstrate that FDG-PET/CT can not only quantify the short lived atherosclerotic inflammatory process, but that identifying these changes are of value in predicting long term atherosclerosis progression. These findings support the current use of FDG-PET in drug efficacy assessment trials, and its potential use to direct future treatment strategies for targeting inflammatory pathways that inhibit atherosclerosis development.

Funding and Disclosures: The Dal-PLAQUE study was funded by F Hoffmann-La Roche Ltd. ZAF has received research grants from Roche, GlaxoSmithKline, Merck, VBL Therapeutics, Novartis, Bristol-Myers Squibb, and Via Pharmaceuticals, and honoraria from Roche. JHFR has received honoraria from Roche. AT has received honoraria from Roche, BMS, and Novartis; and research grants from Merck, BMS, Takeda, Genentech, GlaxoSmithKline, and VBL. DK was previously employed at F Hoffmann-La Roche Ltd and 
received share options. PJ, AA, and VM have no potential conflicts of interest of relevant disclosures to declare.

Dr. Joseph is partly supported by a McMaster University Early Career Investigator award.

Dr. Rudd is part-supported by the NIHR Cambridge Biomedical Research Centre, the British Heart Foundation and the Wellcome Trust. 


\section{References:}

1. Libby P, Ridker PM and Maseri A. Inflammation and atherosclerosis. Circulation. 2002;105:1135-43.

2. Inoue S, Egashira K, Ni W, Kitamoto S, Usui M, Otani K, Ishibashi M, Hiasa K, Nishida K and Takeshita A. Anti-monocyte chemoattractant protein-1 gene therapy limits progression and destabilization of established atherosclerosis in apolipoprotein E-knockout mice. Circulation. 2002;106:2700-6.

3. Emami H and Tawakol A. Noninvasive imaging of arterial inflammation using FDG-PET/CT. Current opinion in lipidology. 2014;25:431-7.

4. Fayad ZA, Mani V, Woodward M, Kallend D, Abt M, Burgess T, Fuster V, Ballantyne CM, Stein EA, Tardif JC, Rudd JH, Farkouh ME, Tawakol A and dal PI. Safety and efficacy of dalcetrapib on atherosclerotic disease using novel non-invasive multimodality imaging (dal-PLAQUE): a randomised clinical trial. Lancet. 2011;378:1547-59.

5. Fayad ZA, Mani V, Woodward M, Kallend D, Bansilal S, Pozza J, Burgess T, Fuster V, Rudd JH, Tawakol A and Farkouh ME. Rationale and design of dal-PLAQUE: A study assessing efficacy and safety of dalcetrapib on progression or regression of atherosclerosis using magnetic resonance imaging and 18F-fluorodeoxyglucose positron emission tomography/computed tomography. Am Heart J. 2011;162:214-221 e2.

6. Rudd JH, Myers KS, Bansilal S, Machac J, Rafique A, Farkouh M, Fuster V and Fayad ZA. (18)Fluorodeoxyglucose positron emission tomography imaging of atherosclerotic plaque inflammation is highly reproducible: implications for atherosclerosis therapy trials. Journal of the American College of Cardiology. 2007;50:892-6.

7. Tawakol A, Migrino RQ, Bashian GG, Bedri S, Vermylen D, Cury RC, Yates D, LaMuraglia GM, Furie K, Houser S, Gewirtz H, Muller JE, Brady TJ and Fischman AJ. In vivo 18F-fluorodeoxyglucose 
positron emission tomography imaging provides a noninvasive measure of carotid plaque inflammation in patients. J Am Coll Cardiol. 2006;48:1818-24.

8. Mani V, Itskovich VV, Aguiar SH, Mizsei G, Aguinaldo JG, Samber DD, Macaluso FM and Fayad ZA. Comparison of gated and non-gated fast multislice black-blood carotid imaging using rapid extended coverage and inflow/outflow saturation techniques. Journal of magnetic resonance imaging : JMRI. 2005;22:628-33.

9. Hayashi K, Mani V, Nemade A, Aguiar S, Postley JE, Fuster V and Fayad ZA. Variations in atherosclerosis and remodeling patterns in aorta and carotids. Journal of cardiovascular magnetic resonance : official journal of the Society for Cardiovascular Magnetic Resonance. 2010;12:10.

10. Mani V, Woodward M, Samber D, Bucerius J, Tawakol A, Kallend D, Rudd JH, Abt M and Fayad ZA. Predictors of change in carotid atherosclerotic plaque inflammation and burden as measured by 18-FDG-PET and MRI, respectively, in the dal-PLAQUE study. The international journal of cardiovascular imaging. 2014;30:571-82.

11. Taqueti VR, Di Carli MF, Jerosch-Herold M, Sukhova GK, Murthy VL, Folco EJ, Kwong RY, Ozaki CK, Belkin M, Nahrendorf M, Weissleder R and Libby P. Increased microvascularization and vessel permeability associate with active inflammation in human atheromata. Circ Cardiovasc Imaging. 2014;7:920-9.

12. Abdelbaky A, Corsini E, Figueroa AL, Fontanez S, Subramanian S, Ferencik M, Brady TJ, Hoffmann $U$ and Tawakol A. Focal arterial inflammation precedes subsequent calcification in the same location: a longitudinal FDG-PET/CT study. Circ Cardiovasc Imaging. 2013;6:747-54.

13. Ridker PM, Buring JE, Cook NR and Rifai N. C-reactive protein, the metabolic syndrome, and risk of incident cardiovascular events: an 8-year follow-up of 14719 initially healthy American women. Circulation. 2003;107:391-7.

14. Figueroa AL, Abdelbaky A, Truong QA, Corsini E, Macnabb MH, Lavender ZR, Lawler MA, Grinspoon SK, Brady TJ, Nasir K, Hoffmann U and Tawakol A. Measurement of Arterial Activity on 
Routine FDG PET/CT Images Improves Prediction of Risk of Future CV Events. JACC CardiovasC Imaging. 2013;6:1250-9.

15. Marnane M, Merwick A, Sheehan OC, Hannon N, Foran P, Grant T, Dolan E, Moroney J, Murphy S, O'Rourke K, O'Malley K, O'Donohoe M, McDonnell C, Noone I, Barry M, Crowe M, Kavanagh E, O'Connell M and Kelly PJ. Carotid plaque inflammation on 18F-fluorodeoxyglucose positron emission tomography predicts early stroke recurrence. Annals of neurology. 2012;71:70918.

16. Tawakol A, Fayad ZA, Mogg R, Alon A, Klimas MT, Dansky H, Subramanian SS, Abdelbaky A, Rudd JH, Farkouh ME, Nunes IO, Beals CR and Shankar SS. Intensification of statin therapy results in a rapid reduction in atherosclerotic inflammation: results of a multicenter fluorodeoxyglucosepositron emission tomography/computed tomography feasibility study. J Am Coll Cardiol. 2013;62:909-17.

17. LaRosa JC, Grundy SM, Waters DD, Shear C, Barter P, Fruchart JC, Gotto AM, Greten H, Kastelein JJ, Shepherd J, Wenger NK and Treating to New Targets I. Intensive lipid lowering with atorvastatin in patients with stable coronary disease. The New England journal of medicine. 2005;352:1425-35.

18. Cannon CP, Braunwald E, McCabe CH, Rader DJ, Rouleau JL, Belder R, Joyal SV, Hill KA, Pfeffer MA, Skene AM, Pravastatin or Atorvastatin E and Infection Therapy-Thrombolysis in Myocardial Infarction I. Intensive versus moderate lipid lowering with statins after acute coronary syndromes. The New England journal of medicine. 2004;350:1495-504.

19. Schwartz GG, Olsson AG, Abt M, Ballantyne CM, Barter PJ, Brumm J, Chaitman BR, Holme IM, Kallend D, Leiter LA, Leitersdorf E, McMurray JJ, Mundl H, Nicholls SJ, Shah PK, Tardif JC, Wright RS and dal OI. Effects of dalcetrapib in patients with a recent acute coronary syndrome. The New England journal of medicine. 2012;367:2089-99. 
20. Tawakol A, Singh P, Rudd JH, Soffer J, Cai G, Vucic E, Brannan SP, Tarka EA, Shaddinger BC, Sarov-Blat L, Matthews P, Subramanian S, Farkouh M and Fayad ZA. Effect of treatment for 12 weeks with rilapladib, a lipoprotein-associated phospholipase A2 inhibitor, on arterial inflammation as assessed with $18 \mathrm{~F}-$-fluorodeoxyglucose-positron emission tomography imaging. $J$ Am Coll Cardiol. 2014;63:86-8.

21. STABILITY investigators. Darapladib for preventing ischemic events in stable coronary heart disease. The New England journal of medicine. 2014;370:1702-11.

22. O'Donoghue ML, Braunwald E, White HD, Lukas MA, Tarka E, Steg PG, Hochman JS, Bode C, Maggioni AP, Im K, Shannon JB, Davies RY, Murphy SA, Crugnale SE, Wiviott SD, Bonaca MP, Watson DF, Weaver WD, Serruys PW, Cannon CP. Effect of darapladib on major coronary events after an acute coronary syndrome: the SOLID-TIMI 52 randomized clinical trial. Jama. 2014;312:1006-15.

23. Elkhawad M, Rudd JH, Sarov-Blat L, Cai G, Wells R, Davies LC, Collier DJ, Marber MS, Choudhury RP, Fayad ZA, Tawakol A, Gleeson FV, Lepore JJ, Davis B, Willette RN, Wilkinson IB, Sprecher DL and Cheriyan J. Effects of p38 Mitogen-Activated Protein Kinase Inhibition on Vascular and Systemic Inflammation in Patients With Atherosclerosis. JACC Cardiovasc Imaging. 2012;5:91122.

24. Emami H, Vucic E, Subramanian S, Abdelbaky A, Fayad ZA, Du S, Roth E, Ballantyne CM, Mohler ER, Farkouh ME, Kim J, Farmer M, Li L, Ehlgen A, Langenickel TH, Velasquez L, Hayes W and Tawakol A. The effect of BMS-582949, a P38 mitogen-activated protein kinase (P38 MAPK) inhibitor on arterial inflammation: A multicenter FDG-PET trial. Atherosclerosis. 2015;240:490-496. 25. O'Donoghue ML, Glaser R, Cavender MA, Aylward PE, Bonaca MP, Budaj A, Davies RY, Dellborg M, Fox KA, Gutierrez JA, Hamm C, Kiss RG, Kovar F, Kuder JF, Im KA, Lepore JJ, LopezSendon JL, Ophuis TO, Parkhomenko A, Shannon JB, Spinar J, Tanguay JF, Ruda M, Steg PG, Theroux P, Wiviott SD, Laws I, Sabatine MS, Morrow DA. Effect of Losmapimod on Cardiovascular Outcomes 
in Patients Hospitalized With Acute Myocardial Infarction: A Randomized Clinical Trial. JAMA. 2016;315:1591-9.

26. Fihn SD, Gardin JM, Abrams J, Berra K, Blankenship JC, Dallas AP, Douglas PS, Foody JM, Gerber TC, Hinderliter AL, King SB, 3rd, Kligfield PD, Krumholz HM, Kwong RY, Lim MJ, Linderbaum JA, Mack MJ, Munger MA, Prager RL, Sabik JF, Shaw LJ, Sikkema JD, Smith CR, Jr., Smith SC, Jr., Spertus JA, Williams SV, Anderson JL. 2012 ACCF/AHA/ACP/AATS/PCNA/SCAI/STS guideline for the diagnosis and management of patients with stable ischemic heart disease. Circulation. 2012;126:e354-471.

27. Stone NJ, Robinson JG, Lichtenstein AH, Bairey Merz CN, Blum CB, Eckel RH, Goldberg AC, Gordon D, Levy D, Lloyd-Jones DM, McBride P, Schwartz JS, Shero ST, Smith SC, Jr., Watson K, Wilson PW, Eddleman KM, Jarrett NM, LaBresh K, Nevo L, Wnek J, Anderson JL, Halperin JL, Albert NM, Bozkurt B, Brindis RG, Curtis LH, DeMets D, Hochman JS, Kovacs RJ, Ohman EM, Pressler SJ, Sellke FW, Shen WK, Smith SC, Jr., Tomaselli GF. 2013 ACC/AHA guideline on the treatment of blood cholesterol to reduce atherosclerotic cardiovascular risk in adults. Circulation. 2014;129:S1-45. 28. Ridker PM and Luscher TF. Anti-inflammatory therapies for cardiovascular disease. European heart journal. 2014;35:1782-91.

29. Facey K, Bradbury I, Laking G and Payne E. Overview of the clinical effectiveness of positron emission tomography imaging in selected cancers. Health technology assessment. 2007;11:iii-iv, xi267.

30. Hillner BE, Siegel BA, Hanna L, Duan F, Quinn B and Shields AF. 18F-fluoride PET used for treatment monitoring of systemic cancer therapy: results from the National Oncologic PET Registry. Journal of nuclear medicine : official publication, Society of Nuclear Medicine. 2015;56:222-8.

31. Marcus C, Paidpally V, Antoniou A, Zaheer A, Wahl RL and Subramaniam RM. 18F-FDG PET/CT and lung cancer: value of fourth and subsequent posttherapy follow-up scans for patient 
management. Journal of nuclear medicine : official publication, Society of Nuclear Medicine.

2015;56:204-8. 
Table 1: Baseline Participant Characteristics:

\begin{tabular}{|c|c|c|c|c|}
\hline Characteristic: & $\begin{array}{l}\text { Participants } \\
\text { in Dal-Plaque } \\
(\mathrm{N}=130)\end{array}$ & $\begin{array}{l}\text { Participants } \\
\text { in current } \\
\text { study } \\
(\mathrm{N}=42)\end{array}$ & $\begin{array}{l}\text { Participants } \\
\text { excluded } \\
\text { from current } \\
\text { study } \\
(\mathrm{N}=88)\end{array}$ & $\mathrm{p}$-value \\
\hline \multicolumn{5}{|l|}{ Clinical Characteristics } \\
\hline Age, years; Mean (SD) & $63.6(8.1)$ & $62.5(8.3)$ & $64.2(7.9)$ & 0.27 \\
\hline Female, N(\%) & $\begin{array}{l}24 / 130 \\
(0.18)\end{array}$ & $12 / 42(0.28)$ & $12 / 88(0.14)$ & 0.07 \\
\hline Current smoker, N(\%) & $\begin{array}{l}17 / 130 \\
(0.13)\end{array}$ & $5 / 42(0.12)$ & $12 / 88(0.14)$ & 1.00 \\
\hline $\begin{array}{l}\text { Treatment with active } \\
\text { study medication } \\
\text { (dalcetrapib) }\end{array}$ & $\begin{array}{l}64 / 130 \\
(0.49)\end{array}$ & $24 / 42(0.57)$ & $40 / 88(0.46)$ & 0.28 \\
\hline $\begin{array}{l}\text { History of coronary heart } \\
\text { Disease, } \mathrm{N}(\%)\end{array}$ & $\begin{array}{l}111 / 130 \\
(0.85)\end{array}$ & $76 / 88(0.86)$ & $35 / 42(0.83)$ & 0.85 \\
\hline Diabetes, N(\%) & $\begin{array}{l}39 / 130 \\
(0.30)\end{array}$ & $14 / 42(0.33)$ & $25 / 88(0.28)$ & 0.71 \\
\hline Hypertension, N(\%) & $\begin{array}{l}95 / 130 \\
(0.73)\end{array}$ & $30 / 42(0.71)$ & $65 / 88(0.74)$ & 0.93 \\
\hline SBP, mmHg; mean (SD) & $121.4(15.4)$ & $117.3(14.3)$ & $123.4(15.6)$ & 0.03 \\
\hline
\end{tabular}




\begin{tabular}{|c|l|l|l|l|}
\hline LDL, mg/dL; mean (SD) & $74.2(21.0)$ & $71.2(20.1)$ & $75.6(21.4)$ & 0.25 \\
\hline HDL, mg/dL; mean (SD) & $44.4(13.5)$ & $46.9(13.8)$ & $43.2(13.3)$ & 0.15 \\
\hline BMI, kg/m²; mean (SD) & $29.7(5.5)$ & $28.4(4.3)$ & $30.3(6.0)$ & 0.04 \\
\hline $\begin{array}{c}\text { eGFR, ml/min/1.73 m²; } \\
\text { mean (SD) }\end{array}$ & $76.4(12.9)$ & $77.0(11.7)$ & $76.1(13.5)$ & 0.69 \\
\hline $\begin{array}{c}\text { Imaging Characteristics } \\
\text { Magnetic resonance imaging: }\end{array}$ & $1.1(0.2)$ & $1.2(0.2)$ & 0.02 \\
\hline $\begin{array}{c}\text { Baseline mean carotid } \\
\text { wall thickness (mm) }\end{array}$ & $1.2(0.2)$ & $26.3(6.7)$ & $30.6(8.9)$ & 0.01 \\
\hline $\begin{array}{c}\text { Baseline mean carotid } \\
\left.\text { wall area (mm }{ }^{2}\right)\end{array}$ & $28.8(8.3)$ & $1.9(0.4)$ & $2.1(0.5)$ & 0.04 \\
\hline $\begin{array}{l}\text { Positron Emission Tomography: } \\
\text { Baseline TBR of most } \\
\text { diseased segment of the } \\
\text { carotid artery }\end{array}$ & $2.0(0.5)$ & & \\
\hline
\end{tabular}

$\mathrm{BMI}=$ body mass index, eGFR $=$ estimated glomelular filtration rate, $\mathrm{FDG}=18$ fluorodeoxyglucose, $\mathrm{HDL}=$ high density lipoprotein, $\mathrm{LDL}=$ low density lipoprotein, $\mathrm{SBP}=$ systolic blood pressure, $\mathrm{SD}=$ standard deviation 
Table 2: Changes in Atherosclerosis Progression over 24 Months in Participants With or Without Increased Arterial Inflammation at 6 Months

\begin{tabular}{|c|c|c|c|c|c|c|}
\hline \multirow[b]{2}{*}{ Study Group } & \multicolumn{3}{|c|}{ Percent Change in MWT } & \multicolumn{3}{|c|}{ Percent Change in MWA } \\
\hline & $\begin{array}{l}\text { mean } \\
(\mathrm{SE})\end{array}$ & $\begin{array}{l}\text { P-value for } \\
\text { within- } \\
\text { group } \\
\text { change } \\
\text { from } \\
\text { baseline }\end{array}$ & $\begin{array}{c}\text { P-value } \\
\text { between- } \\
\text { group } \\
\text { comparison }\end{array}$ & $\begin{array}{l}\text { mean } \\
\text { (SE) }\end{array}$ & $\begin{array}{l}\text { P-value } \\
\text { for } \\
\text { change } \\
\text { from } \\
\text { baseline }\end{array}$ & $\begin{array}{l}\text { P-value } \\
\text { between- } \\
\text { group } \\
\text { comparis } \\
\text { on }\end{array}$ \\
\hline $\begin{array}{c}\text { All } \\
\text { Participants }\end{array}$ & $\begin{array}{l}8.49 \\
(3.80)\end{array}$ & 0.013 & NA & $\begin{array}{l}12.98 \\
(4.98)\end{array}$ & 0.031 & NA \\
\hline $\begin{array}{l}\text { Increased } \\
\text { FDG uptake } \\
\qquad(\mathrm{N}=18)\end{array}$ & $\begin{array}{l}17.49 \\
(7.19)\end{array}$ & 0.026 & 0.027 & $\begin{array}{l}25.50 \\
(9.77)\end{array}$ & 0.018 & 0.038 \\
\hline $\begin{array}{l}\text { No increased } \\
\text { FDG uptake } \\
(\mathrm{N}=24)\end{array}$ & $\begin{array}{c}1.74 \\
(3.42)\end{array}$ & 0.616 & & $\begin{array}{l}3.59 \\
(3.95)\end{array}$ & 0.373 & \\
\hline
\end{tabular}

$\mathrm{FDG}=18$-fluorodeoxyglucose. $\mathrm{MRI}=$ Magnetic Resonance Imaging. $\mathrm{MWT}=$ mean wall thickness. MWA = mean wall area. SE = standard error 
Table 3. Atherosclerosis Progression in Participants with and without Increased

\section{$\underline{\text { Arterial Inflammation }}$}

\begin{tabular}{|c|c|c|c|c|}
\hline Endpoints & Model & Estimate (SE) & $\begin{array}{c}\text { Adjusted } R^{2} \text { of } \\
\text { Model }\end{array}$ & p-value \\
\hline Increased & Univariate Model & $15.75(7.36)$ & 0.08 & 0.038 \\
\hline $\begin{array}{l}\text { arterial } \\
\text { inflammation }\end{array}$ & $\begin{array}{l}\text { Multivariable } \\
\text { Model } 1\end{array}$ & $20.81(8.10)$ & 0.08 & 0.014 \\
\hline $\begin{array}{l}\text { at } 6 \text { months } \\
\text { vs. } \Delta \text { MWT at } \\
24 \text { months }\end{array}$ & $\begin{array}{l}\text { Multivariable } \\
\text { Model } 2\end{array}$ & 14.44 (5.86) & 0.50 & 0.018 \\
\hline Increased & Univariate Model & $21.91(9.58)$ & 0.09 & 0.027 \\
\hline $\begin{array}{l}\text { arterial } \\
\text { inflammation }\end{array}$ & $\begin{array}{l}\text { Multivariable } \\
\text { Model 1* }\end{array}$ & $25.81(10.76)$ & 0.06 & 0.022 \\
\hline $\begin{array}{l}\text { at } 6 \text { months } \\
\text { vs. } \Delta \text { MWA at } \\
24 \text { months }\end{array}$ & $\begin{array}{l}\text { Multivariable } \\
\text { Model } 2^{* * *}\end{array}$ & $22.80(7.56)$ & 0.52 & 0.004 \\
\hline
\end{tabular}

${ }^{*}$ Covariates: Age, gender, treatment allocation. ${ }^{* *}$ Covariates: PAI1 activity, baseline MWT on MRI. ${ }^{* * *}$ Covariates: PAI1A0, baseline MWA on MRI. Note: MWT and MWA were strongly correlated, therefore baseline MWT was used as a covariate in the analysis examining changes in MWT and baseline MWA was used in the analysis examining changes in MWA. $\mathrm{BMI}=$ body mass index, $\mathrm{FDG}=\mathrm{FDG}=18$-fluorodeoxyglucose, $\mathrm{MRI}=$ Magnetic Resonance 
Imaging, MWT $=$ mean wall thickness, MWA $=$ mean wall area, PAI1 $=$ Plasminogen activator inhibitor-1, PET = positron emission tomography.

\section{Figure Legend:}

Figure 1: Percent Change in Carotid (A) Mean Wall Thickness and (B) Mean Wall Area at 24 Months in Participants With or Without Evidence of Increased Arterial Inflammation at 6 Months
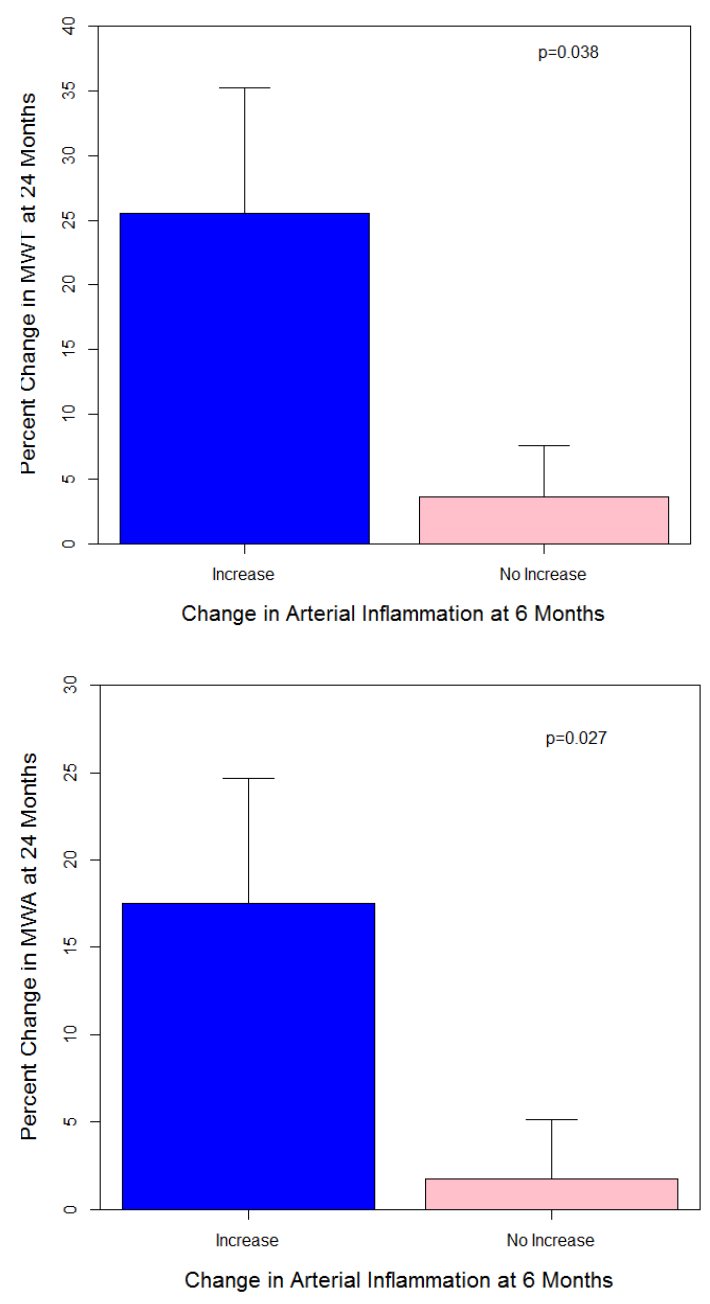
Figure 2: Changes in Arterial Inflammation in Groups with the Greatest Amount of Atherosclerosis Progression Compared to Less Progression Measured by (A) Mean Wall Thickness and (B) Mean Wall Area
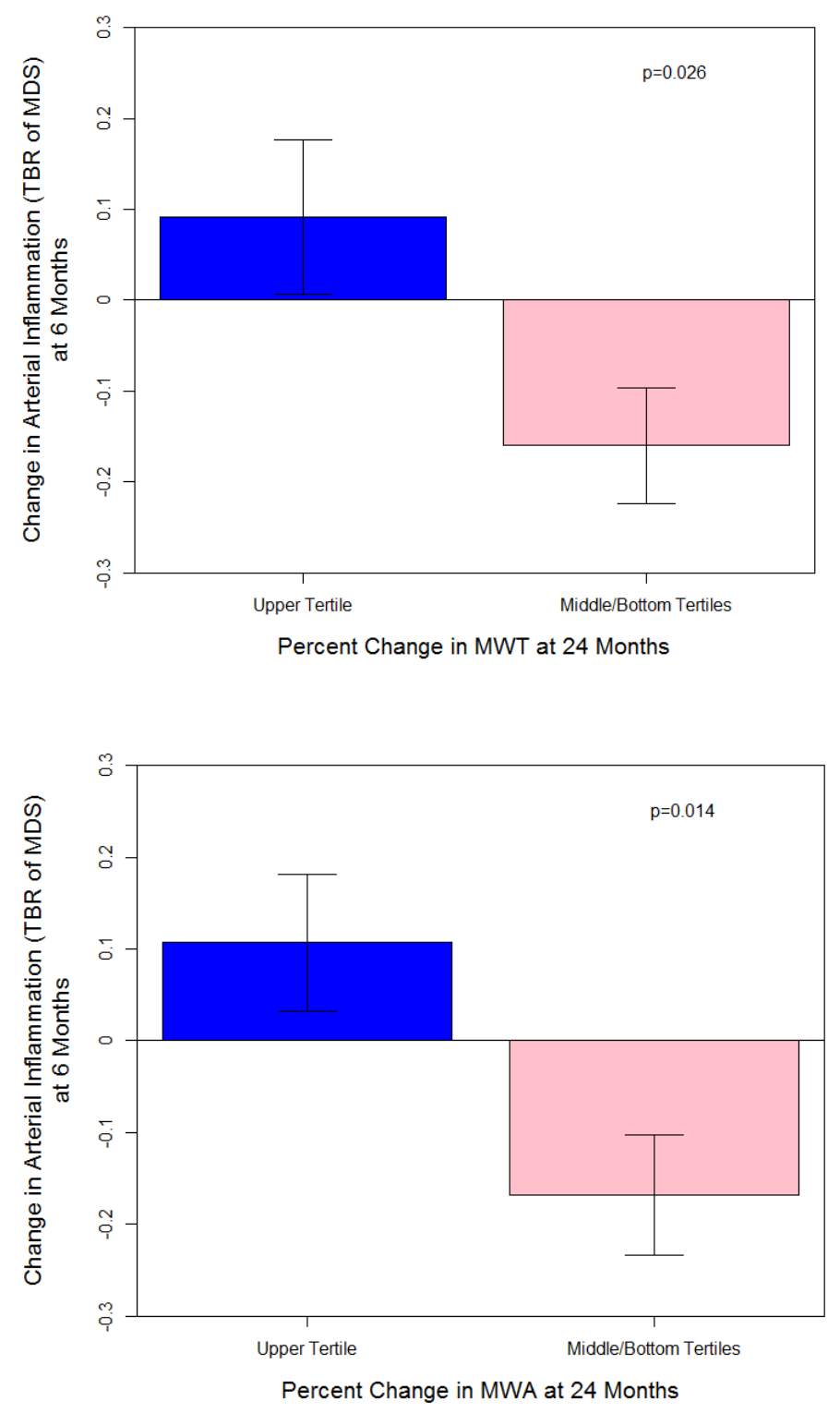